\title{
Clinical Application of Recombinant Human Endostatin in Postoperative Early Complementary Therapy on Patients with Non-small Cell Lung Cancer in Chinese Mainland
}

\author{
Qiang Zhu' ${ }^{1}$, Qi Zang ${ }^{1 *}$,Zhong-Min Jiang ${ }^{1}$, Wei Wang ${ }^{1}$, Ming Cao ${ }^{1}$, Gong-Zhang \\ $\mathrm{Su}^{1}$, Tian-Chang Zhen ${ }^{1}$, Xiao-Tian Zhang ${ }^{2}$, Ning-Bo Sun ${ }^{2}$, Cheng Zhao ${ }^{3}$
}

\begin{abstract}
Objective: To explore the clinical application of recombinant human endostatin (Endostar) in the treatment of patients with non-small cell lung cancer (NSCLC) in Chinese mainland. Materials and Methods: A total of 75 patients diagnosed as NSCLC were randomly divided into control group (37 cases) and treatment group (38 cases). Control group was treated with postoperative complementary chemotherapy containing two-agent platinum protocol on postoperative $\mathrm{d} 21,3$ weeks as a cycle, for totally $4 \sim 6$ cycles. On this basis, treatment group was added with Endostar $7.5 \mathrm{mg} / \mathrm{m}^{2}$ on postoperative d8 9, 3 4 h/time, qd, 14 weeks as a cycle, for totally 4 cycles. The interval between every two cycles was $7 \mathrm{~d}$. The 5-year progression-free survival (PFS), 5-year survival time and complications in both groups were observed. Results: Compared with control group, the average PFS increased evidently in treatment group by 9.8 months (41.6 months $v s .31 .8$ months), and there was significant difference $(P<0.05)$. And the median PFS was 42.5 months in treatment group, obviously longer than that in control group (33.7 months) by 8.8 months $(P<0.05)$. Additionally, the 5-year overall survival rate (OS), average survival time and median survival time (MST) were 47.4\%, 50.1 months and 59.3 months in treatment group, significantly higher than the $29.7 \%, 42.1$ months and 43.5 months in control group $(P<0.05)$. Only 1 patient showed poor healing of surgical wound in treatment group, but no surgery-associated complication was found in control group. Moreover, the postoperative complementary therapy-connected complication rates were 63.2\% (24/38) and 59.5\% (22/37) in treatment group and control group respectively, but there was no significant difference $(P>0.05)$. Conclusions: The application of Endostar combined with sensitive platinum-contained chemotherapeutic agents in the postoperative complementary chemotherapy can be widely used in clinic because it can significantly prolong the long-term survival time of patients with NSCLC.
\end{abstract}

Keywords: Non-small cell lung cancer - recombinant human endostatin - postoperative complementary chemotherapy - progression-free survival - overall survival rate

Asian Pac J Cancer Prev, 16 (9), 4013-4018

\section{Introduction}

Lung cancer is one of the most common malignant tumors all over the whole world, whose mortality ranks the first among various malignant tumors (Blobocan, 2012), in which non-small cell lung cancer (NSCLC) accounts for $80 \%$ (Wu et al, 2010). Postoperative complementary chemotherapy following radical surgery has become the basic therapeutic protocol for patients with early and moderate NSCLC. However, the survival time of NSCLC patients is still low after surgery. How to improve the longterm survival time and the quality of life (QOL) of NSCLC patients has become a tough issue for physicians in clinic. In recent years, the application of anti-angiogenesis agents has become a new hot-topic in the treatment of NSCLC patients, in which recombinant human endostatin,
Endostar (YH-16), has proved its favorable efficacy in treating advanced NSCLC patients when combined with chemotherapeutic agents. Endostar can not only increase the clinical efficacy of chemotherapeutic agents, but also significantly prolong the disease progression time and improve the QOL of the patients (Wang et al, 2005; Rong et al., 2012). On this basis, this study group hypothesized whether Endostar could be used in the postoperative complementary therapy so as to prolong the survival time of NSCLC patients. Therefore, from October $8^{\text {th }}$, 2007 to March $15^{\text {th }}, 2009$, this group attempted to apply Endostar as a postoperative complementary therapy early after radical surgery for NSCLC patients, and other patients with single postoperative complementary therapy were set as control, whose results demonstrated that the median progression-free survival time (PFS) and

${ }^{2}$ Weifang Medical University, Weifang, ${ }^{~}$ Department of Thoracic Surgery, The Affiliated Qianfoshan Hospital of Shandong University, Jinan, ${ }^{3}$ Shandong University School of Medicine, China*For correspondence:qzang2005@sina.com 
long-term survival rate were significantly prolonged with favorable efficacy obtained after Endostar treatment. This study analyzed the clinical data of those patients, aiming to explore the clinical application value of Endostar combined with chemotherapy in the postoperative early complementary therapy in the treatment of NSCLC patients.

\section{Materials and Methods}

\section{General data}

All patients in this study signed the informed consent forms and this study was approved by the Institutional Review Board of this hospital.

Inclusion criteria: (1)Patients without neo-adjuvant therapy before operation; (2)Patients who received preoperative chest and abdominal computed tomography (CT), bone scanning and coronal magnetic resonance image (MRI), and without distant metastasis; (3)Patients without history of primary tumors in other locations; (4) Patients who received radical surgery and systematic nodal dissection (pulmonary lobectomy, bronchial sleeve lobectomy or pneumonectomy+hilar and mediastinal lymphadenectomy); (5)Patients who were diagnosed as stage IB IIIA by postoperative pathology, according to the Union for International Cancer Control (UICC) TNM stages for Lung in 2009 (version 7); (6)Patients whose postoperative performance status (PS) scale was 0 2 scores; (7)Patients whose heart, liver, kidney and bone marrow functions were tolerable to the therapies; (8)The resected tumor samples from those patients showed sensitivity to the chemotherapeutic agents by drug sensitive test.

Exclusion criteria: The included patients with the following conditions could quit the clinical research: (1)Patients who had poor compliance and could not finish the therapeutic protocols on time; (2) Patients who had intolerable therapy-associated adverse responses,

Table 1. Clinical Characteristic of the NSCLC Patients

\begin{tabular}{lcc}
\hline Characteristics & \multicolumn{2}{c}{ Cases (\%) } \\
\cline { 2 - 3 } & Treatment group & Control group \\
\hline Total & 38 & 37 \\
Age (years) & $63.5(42 \sim 73)$ & $61(45 \sim 70)$ \\
Median (min, max) & & \\
Gender & $30(78.9)$ & $27(73.0)$ \\
Male & $8(21.1)$ & $10(27.0)$ \\
Female & 29 & 29 \\
Types of resection & 6 & 4 \\
Lobectomy & 3 & 4 \\
Sleeve lobectomy & & \\
Pneumonectomy & 2 & 2 \\
Postsurgical stage & 10 & 17 \\
Stage IB & 15 & 10 \\
Stage IIA & 11 & 20 \\
Stage IIB & 19 & 15 \\
Stage IIIA & 19 & \\
Histology type & & \\
Squamous carcinoma & & \\
Adenocarcinoma & & \\
Others & & \\
\hline
\end{tabular}

like severe hematology, gastrointestinal toxicity and hepatorenal function damage; (3)Patients who were insensitive to chemotherapeutic agents.

Grouping: According to the above criteria, a total of 75 patients were selected and randomly divided into treatment group and control group. In treatment group, there were 38 patients, in whom there were 30 males and 8 females; aged 42 73 years, with median age of $63.5 ; 29$ were with pulmonary lobectomy, 6 with pulmonary bronchial sleeve lobectomy and 3 with pneumonectomy; postoperative pathological stage: 2 in stage IB, 13 in IIA and 15 in IIB and 8 in IIIA; and histocytological patterns: 19 were with squamous cellular carcinoma, 18 with adenocarcinoma and 1 with other pattern. In control group, there were 37 patients, in whom there were 27 males and 10 females; aged 45 70 years, with median age of $61 ; 29$ were with pulmonary lobectomy, 4 with pulmonary bronchial sleeve lobectomy and 4 with pneumonectomy; postoperative pathological stage: 4 in stage IB, 15 in IIA and 12 in IIB; and histocytological patterns: 20 were with squamous carcinoma, 15 with adenocarcinoma and 2 with other patterns. There was no significant difference between two groups in clinical characteristics (gender, age, surgical method, pathological stage and histocytological patterns) when included $(P<0.05)$, as shown in Table 1 .

\section{Therapeutic protocols}

Control group: Two-agent platinum protocol was given as the postoperative complementary chemotherapy on postoperative $\mathrm{d} 21$. The chemotherapeutic agents were determined according to the drug sensitive test results of the resected tumor samples. The combination and dosage of chemotherapeutic agents referred to the National Comprehensive Cancer Network (NCCN) Guidelines for Clinical Practice of Tumors (edition 2007): cisplatin 75 $\mathrm{mg} / \mathrm{m}^{2}$, d 2 4, i.v.; carboplatin AUC5, d1, i.v.; docetaxel $175 \mathrm{mg} / \mathrm{m}^{2}$, d1, i.v.; gemcitabine $1000 \mathrm{mg} / \mathrm{m}^{2}$, d1 and 8 , i.v.; pemetrexed $500 \mathrm{mg} / \mathrm{m}^{2}$, d1, i.v.; and vinorelbine 25 $\mathrm{mg} / \mathrm{m}^{2}, \mathrm{~d} 1$ and 8 , i.v., 3 weeks as a cycle, for totally $4 \sim 6$ cycles.

Treatment group: On the basis of treatment in control group, Endostar (Shandong Yantai Maidejin Biological Engineering Co., Ltd) $7.5 \mathrm{mg} / \mathrm{m}^{2}$ was diluted into $250 \mathrm{~mL}$ normal saline and intravenously injected on postoperative d8 9, 3 4 h/time, q.d., 14 weeks as a cycle, for totally 4 cycles. The interval between every two cycles was $7 \mathrm{~d}$. Patients with recurrence in both groups were given support therapy with regulated chemotherapeutic protocols and local radiotherapy, etc.

\section{Evaluation of follow-up efficacy}

(1)Postoperative average progression-free survival (PFS), median survival time (MST) and overall survival (OS) rate were observed. PFS was defined as the time period from the postoperative $1 \mathrm{~d}$ to postoperative recurrence or metastasis, while OS as the surgical day to the terminal follow-up day or death. PFS and OS were expressed by months. The amount of patients still survived at the end of follow up was regarded as censored data. (2) Complications like bronchopleural fistula and surgical wound healing condition were observed. 
Table 2. Comparison of Complications between Two Groups [n (\%)]

\begin{tabular}{|c|c|c|c|}
\hline \multicolumn{2}{|c|}{ Complications } & Treatment group $(n=38)$ & Control group $(n=37)$ \\
\hline \multirow[t]{2}{*}{ Surgery-associated complications } & Poor healing of surgical wound & $1(2.6)$ & $0(0.0)$ \\
\hline & Bronchopleural fistula & $0(0.0)$ & $0(0.0)$ \\
\hline \multirow{8}{*}{$\begin{array}{l}\text { Postoperative complementary } \\
\text { therapy-connected complications }\end{array}$} & Nausea and vomiting & $3(7.9)$ & $4(10.8)$ \\
\hline & Hepatic dysfunction & $4(10.5)$ & $3(8.1)$ \\
\hline & Myelosuppression & $3(7.9)$ & $3(8.1)$ \\
\hline & Alopecia & $2(5.3)$ & $1(2.7)$ \\
\hline & Fatigue & $6(15.8)$ & $5(13.5)$ \\
\hline & Soreness and pain of joints & $5(13.2)$ & $1(2.7)$ \\
\hline & Adverse cardiac events & $1(2.6)$ & $5(13.5)$ \\
\hline & Total & $24(63.2)$ & $22(59.5)$ \\
\hline
\end{tabular}

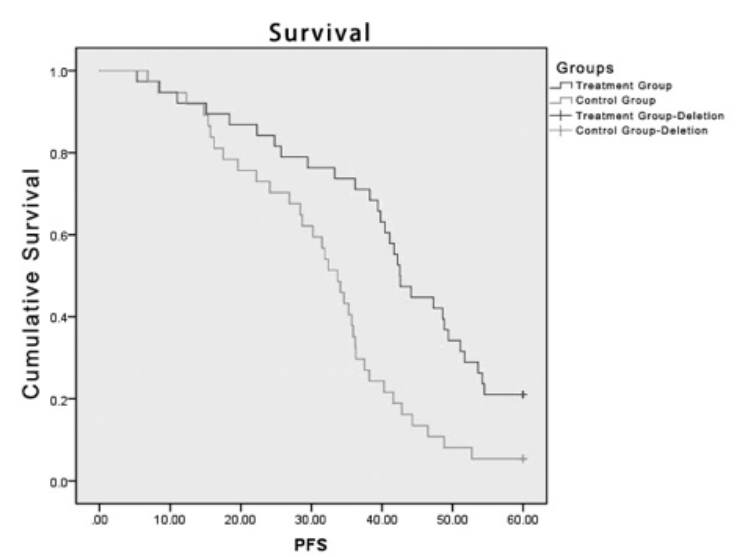

Figure 1. Comparison of 5-year PFS between Two Groups

\section{Follow-up}

The follow up was conducted by outpatient reexamination or phone calls and ended on March $16^{\text {th }}, 2014$. The follow-up contents included medical history, physical examination and chest and abdominal CT. Brain CT or MRI and bone scanning was performed if the patients had corresponding symptoms. Follow-up frequency: 1 time/ 3 months in the first 2 years after operation, and then 1 time/6 month until the postoperative 5 years.

\section{Statistical data analysis}

SPSS17.0 software was applied for all data analysis. Enumeration data comparison was expressed by percentage (\%) and analyzed with $\chi^{2}$ test. DFS and OS were calculated by Kaplan-Meier and survival time was compared by Log-rank test. $P<0.05$ was considered to be significant.

\section{Results}

\section{Comparison of 5-year PFS between two groups}

Compared with control group, the average PFS increased evidently in treatment group by 9.8 months (41.6 months vs. 31.8 months), and there was significant difference $(P<0.05)$. And the median PFS was 42.5 months in treatment group, which was obviously longer than control group (33.7 months) by 8.8 months $(P<0.05)$, as shown in Figure 1.

\section{Comparison of 5-year OS between two groups}

The 5-year overall survival rate (OS), average survival time and median survival time (MST) were 47.4\%, 50.1

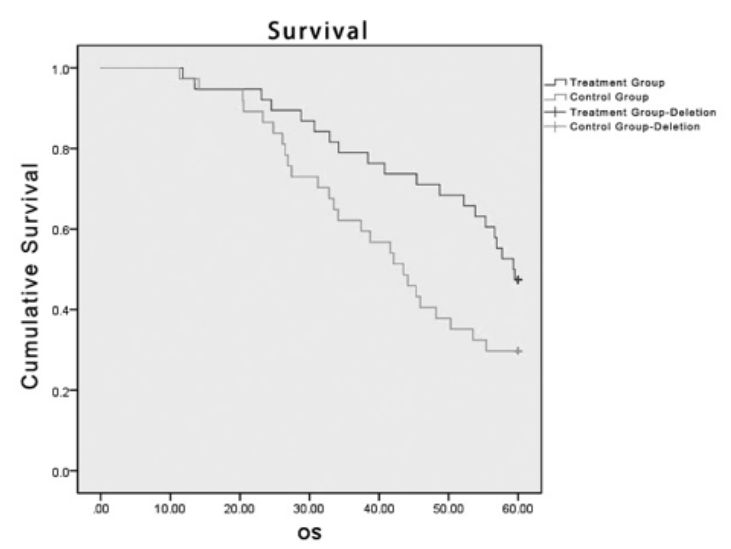

Figure 2. Comparison of 5-year OS between Two Groups

months and 59.3 months in treatment group, significantly higher than the $29.7 \%, 42.1$ months and 43.5 months in control group $(P<0.05)$, as shown in Figure 2.

\section{Comparison of complications between two groups}

Surgery-associated complications: Only 1 patient showed poor healing of surgical wound in treatment group, and no surgery-associated complication was found in control group. Postoperative complementary therapy-connected complications: The complications mainly included nausea and vomiting, hepatic dysfunction, myelosuppression, alopecia, fatigue, soreness and pain of joints and muscles and adverse cardiac events, whose rates were $63.2 \%(24 / 38)$ and $59.5 \%(22 / 37)$ in treatment group and control group, respectively, but there was no significant difference $(P>0.05)$.

\section{Discussion}

With the continuous enhancement of human healthy concept and the development of medical diagnostic techniques, the diagnostic rate of lung cancer is increasing annually. However, the long-term survival rate of lung cancer patients has not been significantly improved (Lu et al., 2013; Yan et al., 2013; Hou et al., 2014; Ji et al., 2014). The 5-year survival rate of NSCLC patients undergoing surgeries is only $30 \% \sim 40 \%$ in the maximum, with the cause of death being tumor recurrence and metastasis, thus leading to the unsatisfactory clinical outcomes (Fei et al., 2013; Cui et al., 2014; Huang et al., 2014). How to reduce the postoperative recurrence and metastasis so as to prolong the survival time and improve 
the QOL of NSCLC patients has become a serious issue for clinical physicians in practices. In the development of malignant tumors, the tumor cells can early spread and survive in blood circulation system, lymph node and bone marrow as well as various tissues and organs, thus forming the sub-clinical "micro-metastatic nidi". Although the primary nidi can be completely excised and lymph node be systematically removed through modified surgeries, adelomorphic micro-metastatic nidi of tumors have already been formed, which are the origins and basis for the postoperative recurrence and metastasis of NSCLC patients (Rosell et al., 2001; Felip et al., 2002). Surgical resection itself is also an important factor for the postoperative recurrence and metastasis of NSCLC. On one hand, surgical operations can lead to the spread of tumor cells into blood circulation, thus triggering the postoperative recurrence and metastasis of tumors; on the other hand, most of the postoperative residual tumor cells develop apoptosis in vivo while few of them in dormant state (stage $\mathrm{G}_{0}$ ) and only a small amount have proliferation. In addition, surgeries and anesthesia may bring about serious trauma and influence on human bodies, thus leading to the decrease of immunology, changes of tumor inhibitors in blood, increase of locoregional blood volume in micro-metastatic nidi of tumors and improve the neovascularization, the changes of which can release the inhibited tumor cells and activate the tumor cells in dormant state, thus resulting in the continuous proliferation of cells and then developing into significant dominant recurrence and metastasis of tumors in clinic.

In 1971, Folkman initially proposed the theory of tumor angiogenesis and the therapeutic conceive and mechanism for anti-angiogenesis (Folkman, 1971). Neovascularization is a precondition for the growth and invasion of tumors, and also a morphological basis for the proliferation and metastasis of tumors. When the tumor size was $>2 \mathrm{~mm}^{3}$, the survival and growth of tumors mainly depend on the neovascularization. If there is no neovascularization, the tumor tissues will degenerate. Once neovascularization is formed, the tumor will grow rapidly. The neovascularization not only provides nutrition for tumor cells, but also offers channels for the metastasis of tumor cells. The formation of neonatal vessels is a required way for the growth and development of tumors, so blocking the neonatal vessels of micro-metastatic nihi is critical to prevent the postoperative recurrence and metastasis of NSCLC patients.

In recent years, Avastin, Cetuximab, Pazopanib and Endostatin, etc., are the most commonly used antiangiogenesis drugs in clinic, in which Endostain, as an endogenous glycoprotein, was initially extracted from the mice angioblast tumor stain culture solution, and is effective in anti-angiogenesis. In 2005, new-type recombinant human Endostatin, Endostar, was approved by the State Food and Drug Administration of China for NSCLC patients. Endostar can target $>12 \%$ angiogenesis regulation genes in human genomes, inhibit the metastasis, adhesion and growth of vascular endothelial cells (VEC) and the revascularization, and directly influence the apoptosis of VEC so as to directly play the antiangiogenesis function. Moreover, it can also exert the anti-angiogenesis effect by multiple targets via regulating the vascular endothelial growth factor (VEGF) on the surface of tumor cells, thus leading to the dormancy or atrophy of tumors indirectly (Folkman, 2006).

Increasing more animal and clinical experiments demonstrated that Endostar could inhibit the progression and metastasis of multiple tumors (Calvo et al, 2002; Tsunemi et al., 2003; Xia et al., 2003; Yao et al., 2004; Te Velde et al., 2005; Kaya et al., 2007). Wang et al (Wang et al., 2011) established the animal models with idiopathic lung metastasis of Lewis cellular carcinoma in mice, whose observational results indicated that Endostar could effectively inhibit the progression of the distant metastatic nidi after the resection of the primary tumor nidi. The latest research revealed that Endostar concomitant with cisplatin could not only effectively suppress the cell growth of lung cancers in single use, but also reduce the lymph node metastasis by inhibiting the lymphangiogenesis of tumors (Dong et al., 2013). At present, Endostar concomitant with chemotherapeutic agents are believed to have synergistic effect in treating NSCLC patients, and most researches focused on the basic animal experiments and clinical practices on patients with advanced NSCLC (Wang et al., 2005; Wang et al., 2011; Peng et al., 2012; Rong et al., 2012; Dong et al., 2013). However, study on the efficacy of Endostar as the complementary therapy for early NSCLC patients after radical operations is barely seen in clinic. In this study, Endostar was applied early after operation (8 9 $\mathrm{d}$ after operation) as an important part of the postoperative complementary therapies to NSCLC patients, with favorable clinical efficacy obtained after combined with the platinum-included chemotherapeutic agents (administrated in postoperative 3 weeks). This study also found that the average PFS and 5-year survival time were 41.6 months and 50.1 months, with 5-year OS rate being $47.4 \%$, which were all significantly higher in treatment group than in control group, illustrating that Endostar concomitant with platinum-included chemotherapeutic agents could markedly prolong the postoperative survival time and improve the quality of life of NSCLC patients.

In the procession of clinical treatment on NSCLC, the early application of Endostar is of great significance and is effective in biological action. Macpherson et al (Macpherson et al., 2003) suggested that Endostar, which had the ability of inhibiting the tumor angiogenesis and activity, was more effective when applied in the formation phase than in maturation phase of tumor cells, indicating that the application of Endostar in the early tumor angiogenesis might bring about better clinical efficacy. Tatyana et al (Tatyana et al., 2007) compared the early-stage and advanced-stage therapies on mice models with PC, which found that in the early formation stage of tumors, early anti-angiogenesis therapy could inhibit the differentiation process of tumors that might trigger the malnutrition of tumors and prevent the formation of metastatic nidi, thus increasing the patients' survival time obviously. Endostar was applied early (8 9 d after operation) in this study, which could better block the neo-angiogenesis of existed sub-clinical micro-metastatic nidi, reduce the vascular permeability of tumor nidi and damage the immature vessels so as to postpone the 
growth of tumor nidi and impede the tumor cell growth in phase $G_{2}$. In addition, the application of sensitive platinum-included chemotherapeutic agents 3 weeks after operation could further kill the tumor cells and avoid the postoperative complications (poor recovery of the wounds and bronchopleural fistula, etc.) due to the early use of those chemotherapeutic agents.

Additionally, Endostar could also increase the accumulation of peri-vascular cells, which might trigger transient stability of vessels and improve the oxygen transformation in ischemic area when the cells reached to the tumor vessels. In the tumor vessel normalization "time window", the application of chemotherapeutic agents could increase the agent concentration in tumor nidi so as to achieve better therapeutic efficacy (Dass et al., 2008; Huang et al., 2010). At present, the appearing time of tumor vessel normalization "time window" after the utilization of Endostar was still unpredictable (Liu et al., 2012). Some scholars used CT perfusion imaging and hypoxic imaging to study the efficacy of Endostar in the treatment of NSCLC patients in stage II and III and compared with that in control group, which demonstrated that the single application of Endostar for 1 week could significantly improve the blood perfusion and hypoxic condition of tumors, so it was believed that the "time window" for radiotherapy was around 1 week after treatment (Jiang et al., 2012). However, the "time window" for chemotherapy is still undefined. It was thought that the tumor vessel normalization appeared 4 10 after Endostar treatment, during which time the application of chemotherapeutic agents could greatly improve the clinical efficacy. But this time window lasted for a short time, about 3 d (Li et al., 2012). However, other researches held contrary options that Endostar concomitant with chemotherapeutic agents or the application of Endostar after chemotherapeutic agents was the optimal protocol and might obtain better clinical efficacy. Meanwhile, they also believed that the chemotherapeutic agents applied within the tumor vessel normalization "time window" was not the primary mechanism of increasing the clinical efficacy (Peng et al., 2012). In this study, Endostar and postoperative complementary chemotherapeutic agents were timely and regularly given to treatment group according to the patients' administrative time, in which Endostar might be used before, after or simultaneously with the chemotherapeutic agents, thus increasing the administrative rate of the chemotherapeutic agents within the tumor vessel normalization "time window" so as to improve the clinical efficacy. In the mean time, the cytotoxicity of the chemotherapeutic agents might lead to the death of the tumor cells and their requirement to oxygen and nutrition increased (Shojaei et al., 2008). Moreover, Endostar could inhibit the tumor neo-angiogenesis and had direct influence on the nutrition-supply for tumors, thus resulting in the death of sub-clinical metastatic microtumor nidi because of hunger. Endostar had synergetic action with platinum-contained chemotherapeutic agents when used together, which could remarkably improve the therapeutic efficacy of postoperative complementary protocols.

It is commonly believed that Endostar will not increase the chemotherapy-induced adverse responses, but whether it will influence the healing of surgical wounds and bronchial stump and the infection rate of NSCLC patients is still unclear. The previous research found that a longterm injection of Endostar had no severe impact on the wound healing and hepatorenal function (Kirsch et al., 1998), and did not cause weight loss, activity decrease, loss of appetite and opportunistic infection (Sim et al., 1997). The long-term application of anti-angiogenesis agents (such as Bevacizumab) can bring about damage to normal VEC, thus triggering the adverse responses of hypertension, hemorrhage and thrombosis, etc, but they are low in morbidity and slight in severity. However, the influence of those agents on wound healing seems to be more severe. Scappaticci et al (Scappaticci et al., 2005) studied the influence of Bevacizumab on the wound healing of patients with advanced colorectal cancer (CRC). In that study, all patients were divided into two groups, in which one was treated with surgeries 28 60 d before Bevacizumab treatment (group A) and the other with surgeries during the Bevacizumab treatment (group B), and then the above two groups were divided into two sub-groups namely combination group treated with chemotherapy combined with Bevacizumab or single group with single chemotherapy. In group A, the woundhealing associated complication rates in combined group and single group were $1.3 \%$ and $0.5 \%$, respectively. However, in group B, the complication rates were $13.0 \%$ and $3.4 \%$ respectively, indicating that Bevacizumab was effective in blocking the wound healing. Therefore, to prevent the wound-healing associated complications, it is recommended that surgery should be avoid during Bevacizumab combined with chemotherapy, and it is safe to apply Bevacizumab combined with chemotherapy $28 \mathrm{~d}$ after operation. In this study, it was found that Endostar, which was used at early time (8 9 d before operation), did not increase the failure rate of wound healing and the rate of bronchial stump fistula, demonstrating that the postoperative early application of Endostar might be safe, which was predicated to be associated with the facts that Endostar would not increase the above complications and the sample size was small in this study. And whether Endostar can be used to NSCLC patients at earlier time after operation requires further experiment study to prove.

To sum up, this study primarily verified that as to early and moderate NSCLC patients who were able to receive radical surgeries, postoperative early complementary therapy including Endostar and sensitive platinumcontained chemotherapeutic agents could significantly increase the patients' long-term efficacy. It is also believed that with the successful research and development of clinical application of increasingly more multi-target anti-angiogenesis agents, the therapeutic model applied in this study, the postoperative complementary therapy with anti-angiogenesis agents combined with sensitive chemotherapeutic agents on NSCLC patients, will be widely used in clinic.

The small sample size in this study, which may lead to statistical selection bias, can lead to certain limitation in the research results. Therefore, the future clinical study should emphasize on random, allocation concealment, 
double blindness and large scale, and the samples should be followed up in a long term so as to explore the therapeutic cycle, dosage and models of the administrative agents, which will be compared with those in control group, hoping to obtain higher-level research evidences.

\section{References}

Calvo A, Yokoyama Y, Smith LE, et a1 (2002). Inhibition of the mammary carcinoma angiogenic switch in C3 (1)/SV40 transgenic mice by a mutated form of human endostatin. Int $J$ Cancer, 101, 224-34.

Cui L, Liu XX, Jiang Y, et al (2014). Phase II study on dose escalating schedule of paclitaxel concurrent with radiotherapy in treating patients with locally advanced non-small cell lung cancer. Asian Pac J Cancer Prev, 15, 1699-702.

Dass CR, Choong PF (2008). Cancer angiogenesis: targeting the heel of Achilles. Drug Target, 16, 449-54.

Dong X P, Xiao T H, Dong H, et al (2013). Endostar combined with cisplatin inhibits tumor growth and lymphatic metastasis of lewis lung carcinoma xenografts in mice. Asian Pac J Cancer Prev, 14, 3079-83.

Fei ZH, Yao CY, Yang XL, et al (2013). Serum BMP-2 up-regulation as an indicator of poor survival in advanced non-small cell lung cancer patients. Asian Pac J Cancer Prev, 14, 5293-9.

Felip E, Rosell R (2002). Neoadjuvant chemotherapy in non-small cell lung cancer. Curr Med Chem, 9, 893-8.

Folkman J (1971). Tumor angiogenesis: therapeutic implications. $N$ Engl J Med, 285, 1182-6.

Folkman J (2006). Anti-angiogenes in cancer theraphy-endostatin and its mechanisms of action. Expt Cell Res, 312, 594-607.

Globocan 2012: estimated cancer incidence, mortality and prevalence worldwide in 2012. http: //globocan.iarc.fr/Pages/ fact_sheets_cancer.aspx.

Hou ZB, Lu KJ, Wu XL, et al (2014). In vitro and in vivo antitumor evaluation of berbamine for lung cancer treatment.Asian Pac $J$ Cancer Prev, 15, 1767-9.

Huang G, Chen L (2010). Recombinant human endostatin improves anti-tumor efficacy of paclitaxel by normalizing tumor vasculature in Lewis lung carcinoma.J Cancer Res Clin Oncol, 136, 1201-11.

Huang XE, Tian GY, Cao J, et al (2014). Pemetrexed as a component of first-, second- and third- line chemotherapy in treating patients with metastatic lung adenocarcinoma. Asian Pac J Cancer Prev, 14, 6663-7.

Ji ZQ, Huang XE, Wu XY, et al (2014). Safety of Brucea javanica and cantharidin combined with chemotherapy for treatment of NSCLC patients. Asian Pac J Cancer Prev, 15, 8603-5.

Jiang XD, Dai P, Qiao Y, et al (2012). Clinical study on the recombinant human endostatin regarding improving the blood perfusion and hypoxia of non-small-cell lung cancer. Clin Transl Oncol, 14, 437-43.

Lu YY, Huang XE, Cao J, et al (2013). Phase II study on Javanica oil emulsion injection (Yadanzi ${ }^{\circledR}$ ) combined with chemotherapy in treating patients with advanced lung adenocarcinoma. Asian Pac J Cancer Prev, 14, 4791-4.

Kaya M, Wada T, Nagoya S, et al (2007). Prevention of postoperative progression of pulmonary metastases in osteosarcoma by antiangiogenic therapy using endostatin. J Orthop Sci, 12, 562-7.

Kirsch M, Strasser J, Allende R, et al (1998). Angiostatin suppress malignant glioma growth in vivo. Cancer Res, 58, 4654-9.

Li N, Zheng DW, Wei XY, et al (2012). Effects of recombinant human endostatin and its synergy with cisplatin on circulating endothelial cells and tumor vascular normalization in A549 xenograft murine model. J Cancer Res Clin Oncol, 138, 113144.

Liu ZJ, Wang J, Wei XY, et al (2012). Predictive value of circulating endothelial cells for efficacy of chemotherapy with Rhendostatin in non-small cell lung cancer. J Cancer Res Clin Oncol, 138, 927-37.
Macpherson GR, Ng SS, Forbes SL, et al (2003). Anti-angiogenic activity of human endostatin is HIF-1-independent in vitro and sensitive to timing of treatment in a human saphenous vein assay. Mol Cancer Ther, 2, 845-54.

Peng XC, Qiu M, Wei M, et al (2012). Different combination schedules of gemcitabine with endostar affect antitumor efficacy. Cancer Chemother Pharmacol, 69, 239-46.

Rong B, Yang S, Li W, et al (2012). Systematic review and meta-analysis of Endostar (rh-endostatin) combined with chemotherapy versus chemotherapy alone for treating advanced non-small cell lung cancer. World J Surg Oncol, 10, 170.

Rosell R, Felip E, Maestre J, et al (2001). The role of chemotherapy inearly non-small-cell lung cancer management. Lung Cancer, 34, S63-S74

Scappaticci FA, Fehrenbacher L, Cartwright T (2005). Surgical wound healing complications in metastatic colorectal cancer patients treated with bevacizumab. J Surg Oncol, 91, 173-80.

Shojaei F, Ferrara N (2008). Role of the microenvironment in tumor growth and in refractoriness/resistance to anti-angiogenenic therapies. Drug Resist Updat, 11, 219-30.

Sim BKL, O, Reilly MS, Liang H, et al (1997). A recombinant human angiostatin protein inhibits experimental primary and metastatic cancer. Cancer Res, 57, 1329-34.

Tatyana L, Diptiman C, Lisa K, et al (2007). Effects Of sustained antiangiogenic therapy in multistage prostate cancer in TRAMP model. Cancer Res, 67, 5789-97.

Te Velde EA, Reijerkerk A, Brandsma D, et al (2005). Early endostatin treatment inhibits metastatic seeding of murine colorectal cancer cells in the liver and their adhesion to endothelial cells. Br J Cancer, 92, 729-35.

Tsunemi T, Nagoya S, Kaya M, et al (2003). Postoperative progression of pulmonary metastasis in osteosarcoma. Clin Orthop Relat Res, 407, 159-66.

Wang HL, Ning T, Li M, et al (2011). Effect of endostatin on preventing postoperative progression of distant metastasis in a murine lung cancer model. Tumori, 97, 787-93.

Wang JW, Sun Y, Liu YY, et al (2005). Results of randomized, multicenter, double-blind phase III trial of rh-endostatin (YH16) in treatment of advanced non-small cell lung cancer patients. Chin J Lung Cancer, 8, 283-90.

Wu HT, Deng J, Yu SY, et al (2010). The Inhibitory Effects of Rhendostatin (YH-16) in Combination with Radiotherapy on lung adenocarcinoma A549 in Mice and the Underlying Mechanisms. J Huazhong Univ Sci Technol, 30, 108-12.

Xia H, Luo L M, Fan Y F, et al (2003). Inhibitory effect of recombinant human endostatin on angiogenesis and lung metastasis of mouse lung adenocarcinoma, LA795. J First Mil Med Univ, 20 30-3.

Yan HA, Shen K, Huang XE (2013). Clinical study on mannan peptide combined with TP regimen in treating patients with non-small cell lung cancer. Asian Pac J Cancer Prev, 14, 4801-4.

Yao HC, Jin DJ, Sun YN, et al (2004). Endostatin in the treatment of the transplantable model of human laryngeal squamous carcinoma in nude mice. Chinese J Otorhinolaryngol, 39, 394-8. 